\title{
HALLEY'S COMET (Part II): Space Studies
}

\author{
Vassili I. Moroz \\ Space Research Institute \\ Academy of Sciences of USSR \\ Moscow 117810 , USSR
}

\begin{abstract}
ABST'RACT. An international armada of spacecraft encountered Comet Halley in March 1986. The present article gives a brief overview of this unique event during which a cometary nucleus was seen as a spatially resolved object for the first time. It is a very dark body, the shape is irregular and the structure is inhomogeneous; only the sunward side is active. Many parent molecules were identified in the coma, including complicated hydrocarbons. There was also organic matter in the comet dust particles. Extensive studies were made of the complicated plasma environment of the comet.
\end{abstract}

\section{Cometary missions}

An impressive armada of spacecraft encountered Comet Halley in March 1986 (Fig. 1). The largest of them were Vega-1 and Vega-2. They had two goals: (1) studies of Venus by means of balloons and landers and (2) fly-by studies of Comet Halley. In June 1985 both spacecraft successfully delivered the landers and balloons at Venus, made a gravitational swing-by manoeuvre near this planet and were directed towards Comet Halley. The Vega mission was an international project. Although the spacecraft themselves were controlled by the Soviet Union, the scientific programme and the payloads were coordinated by the International Science and Technical Committee (CIST), representing scientific institutions in nine countries. Academician Sagdeev was the president of the CIST.

Also the Giotto project was international. It was set up by the European Space Agency (ESA). Giotto was the first attempt of ESA to visit deep space. The Japanese "PlanetA" project included two nearly identical spacecraft - "Suisei" ("Comet") and "Sakigake" ("Pioneer"). They both studied the solar wind/comet interaction, and Suisei also provided UV-imaging of the hydrogen coma.

Giotto encountered the comet after the two Vega spacecraft. Its trajectory was corrected by the use of accurate data for the comet's orbit which were obtained by Vega. This also became an area of efficient international collaboration. Fast processing, transfer of the information from the Vegas and almost real-time calculations of new ephemerides (taking also into account new ground-based astrometric data) was the subject of the very successful, international "Pathfinder" programme.

Important observations of Comet Halley were provided by two space telescopes in nearEarth orbits (IUE and Astron), sounding rockets and also by the UV-spectrometer from Pioneer Venus orbiter.

How can this remarkable accumulation of space efforts be explained? There are several reasons: 1) Every apparition of Comet Hallev is an important astronomical event because 
this is the brightest periodical comet; its physical properties are more typical of a young comet than of a periodical one; 2) periodical comets are the only ones suitable for close-up studies by spacecraft; 3 ) spacecraft give a unique possibility of approach to the cometary nucleus and to investigate it as a spatially resolved object, 4) cometary nuclei are probably samples of pristine materials and their study opens new paths towards the understanding of the origin of the Solar System.

The main scientific results of the missions to Comet Halley are contained in the Proceedings of several conferences (COSPAR XXVI, Toulouse, 30 June-11 July 1986. Ady. Space Res., Vol. 5 (No. 12), 113-121; ESLAB Symposium on the Exploration of Halley's Comet, Heidelberg, 27-31 October 1986; Symposium on the Diversity and Similarity of Comets, Bruxelles, 6-9 April 1987) and in many journals and papers. There were some special issues on the subject: Nature (Vol. 321, No. 6067, 1986), Kosmicheskije Issledovanija (Vol. 25, No. 5, 1987 - in Russian), Astronomy and Astrophysics (Vol. 187, 1987). The most important topics in these collections of papers are: nucleus, gas, dust and plasma.

Below we will outline the most important achievements, emphasizing those results which give new information about the nucleus. These are the close-up images, the measurements of IR radiation and also the studies of the gas and dust composition which are indirect, but unique sources of data about the chemical composition of the nucleus. The quantity of published original papers is very large and we will include in our references only a small part of them. There are comprehensive reviews devoted to some selected topics with more detailed references, for instance by A'Hearn (1988) about cometary nuclei and by Galeev (1987) about plasma events.

\section{The nucleus: Images and IR-radiometry}

Early in the morning of March 6, 1986, hundreds of people were at their working stations at IKI (Moscow) and at the Soviet Deep Space Network station in Evpatoria (Crimea). The culmination of this huge project was approaching. Vega-1 was about to encounter Comel, Halley.

The nucleus comes nearer and nearer. The comet now looks phantastic. There is a broad cone (fan) with a bright spot at its top and the nucleus inside. The fan is directed towards the Sun, opposite to the orientation of the usual cometary tail. The reason is clear. We see the innermost part of the coma, virtually unattainable for observations before. The cone is formed by dust grains driven out by evaporated volatiles from the sunlit side of the nucleus towards the Sun. Only at a distance of many thousands of kilometers (outside our field of view) the trajectories become curved, due to solar radiation pressure and they reverse their direction, thereby forming the dust tail.

Now there are a few minutes left before the pericenter passage. A huge stone with blurred edges is visible on the displays. This is the nucleus surrounded by dust. Frames follow quickly, one after another; "the stone" is turning before the enchanted audience. It is like a flight above the nucleus on-board an aircraft. The goal has been achieved - for the first time, scientists have seen a cometary nucleus.

The interpretation of these images was not a simple task because of the screening effect by the dust. However, the analysis showed that the true boundaries of the nucleus were visible through the surrounding dust. So the structure, shape, size and reflectivity of the nucleus could be determined.

The nucleus was imaged by the two spacecrafts at different rotation phases of the nucleus and this gives a possibility to reconstruct a $3 \mathrm{D}$ model and to define its size (Fig. 3) and also to evaluate its pericd of rotation and the direction of its axis (Sagdeev et al. 1986a, $1986 \mathrm{~b}, 1986 \mathrm{c})$. 


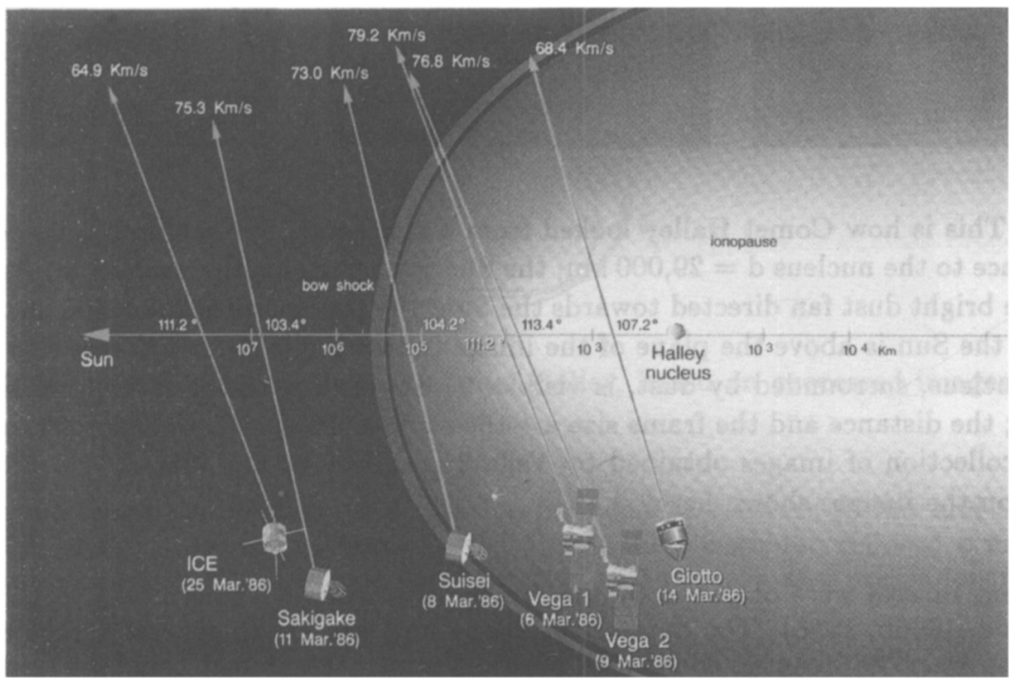

Figure 1: Six spacecrafts met Comet Halley near the descending node of its orbit. Three of them (Vega-1, Vega-2 and Giotto) penetrated deeply into the dust coma. The relative speed of the encounter was $\sim 70-80 \mathrm{kms}^{-1}$ and cometary dust grains were extremely dangerous for the spacecraft; for this reason, protective screens covered the most critical systems by Vega-1 and Vega-2. However, it was decided not to fly too close to the nucleus and minimum distances of 8900 and $8000 \mathrm{~km}$ were chosen. The Giotto spacecraft was more compact and better protected. Its closest approach to the nucleus was only $600 \mathrm{~km}$. 

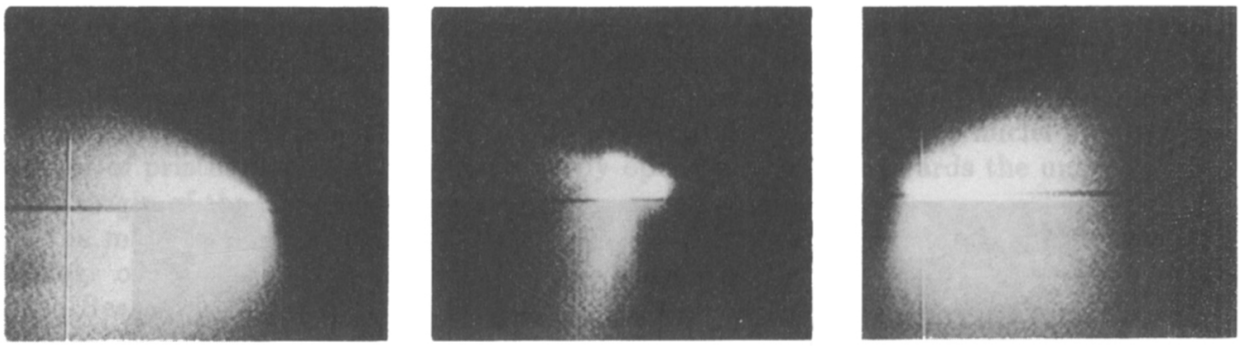

Figure 2: This is how Comet Halley looked from a spacecraft flying through its coma: a) The distance to the nucleus $d=29,000 \mathrm{~km}$; the Sun is on the left, the nucleus is in the right part of the bright dust fan directed towards the Sun; the frame size is $228 \times 308 \mathrm{~km}$. b) d = $8,000 \mathrm{~km}$; the Sun is above the plane of the image (phase angle $\sim 30^{\circ}$ ); frame size $62 \times 84$ $\mathrm{km}$; the nucleus, surrounded by dust, is visible. c) The Sun is to the right, the nucleus is to the left; the distance and the frame size are the same as in a). These examples are taken from the collection of images obtained by Vega-2 (Sagdeev et al., 1986a). No processing was done on the images shown here.

Excellent images were obtained by the Halley Multicolour Camera on Giotto (Keller, et al., 1986a, 1986b, 1988). The data about shape and size are in general agreement with Vega's imagery. The imaging conditions were different: the almost constant (near $100^{\circ}$ ) phase angle compared to the fast change in the phase angle in the case of Vega. This made it possible to combine many Giotto images for contrast enhancement (Fig. 4), but it is impossible to make a $3 \mathrm{D}$ reconstruction using the Giotto data alone.

Let us summarize the main conclusions which result from the studies of the Vega and Giotto images:

1. The nucleus is a single, solid body. Its shape is irregular (like a "potato"); the size is approximately $8 \times 8 \times 16 \mathrm{~km}$ and the volume is $\sim 500 \mathrm{~km}^{3}$.

2. The reflectivity is very low, the geometric albedo is $\sim 0.04$. Thus the nucleus of Comet Halley is one of the darkest bodies in the Solar System.

3. Dust is emitted from the sunlit side of the nucleus only. Its flow is spatially inhomogeneous (and time-variable). There are some regions - a few percent of the entire surface - which eject narrow dust jets, but a weak dust outflow also exists outside these jets. The theory predicted a thin $(\sim 1 \mathrm{~km})$, near-surface layer of dust in which particles are accelerated by the gas from zero to almost final velocity. Now we have evaluated the average optical depth of this layer on the sunlit side of the nucleus to about 0.1 .

4. There is a complicated pattern of surface features, including ring structures, bright spots, etc.

5. The period of rotation of the nucleus is about 2.2 days. Almost the same value was obtained from the analysis of the outburst activity periodicity observed from the Earth (Sekanina and Larson, 1986). The inclination of the equator to the orbital plane is $\sim 30^{\circ}$. The direction of rotation is retrograde, like the orbital motion. Some of the 


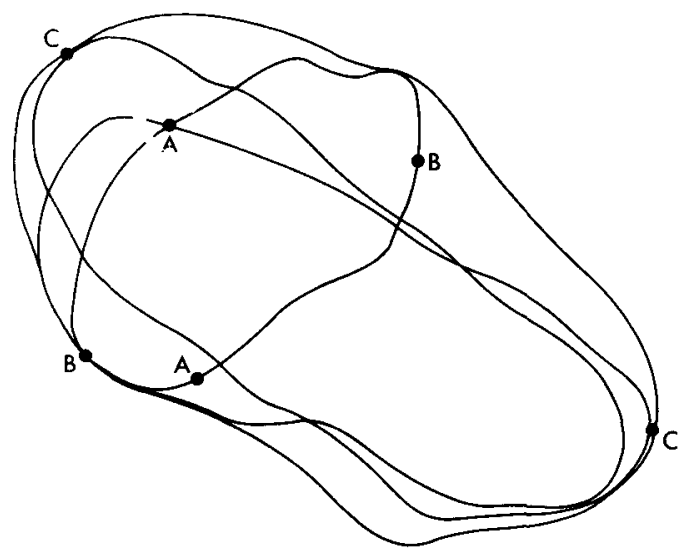

$\mathrm{A}-\mathrm{A}: 7.5 \pm 0.8 \mathrm{~km}$

B - B: $8.2 \pm 0.8 \mathrm{~km}$

C - C: $16.0 \pm 1.8 \mathrm{~km}$

Figure 3: 3D model of the nucleus of Comet Halley, based on processed images obtained by Vega-1 and Vega-2 (Sagdeev et al., 1986 b).

ground-based studies suggest a rotation period near 7.4 days (Millis and Schleicher, 1988) or even 14.6 days (Festou et al., 1988); these authors proposed a model with rotation around the long axis with a period of 14.6 days and precession with a 2.2 day period around another axis inclined by $84^{\circ}$ to the first one.

The TV imaging of the nucleus was supplemented by measurements of its thermal infrared radiation. This was done by the infrared radiometer which was part of the IKS instrument on Vega-1 (Nikolsky et al,, 1987). The result was unexpected: a surface temperature of $\sim 370 \mathrm{~K}$ was estimated in the vicinity of the subsolar point! This fact led to difficulties in understanding the surface structure of the cometary nucleus if we take into account that we need a large amount of water ice there. There is a strong outflow of gas that escapes the nucleus and $\mathrm{H}_{2} \mathrm{O}$ is the main constituent. Consequently, water ice is one of the important components of the nucleus.

To provide the observed flux of $\mathrm{H}_{2} \mathrm{O}$ molecules (see below), a substantial part of the illuminated surface (not less than 25\%) must be icy. A important fraction of the received solar energy is spent on evaporation of this ice. Evaporation leads to cooling and quantitative estimates show that the ice surface temperature in this case should be $\sim 200 \mathrm{~K}$. But the surface is hot and black. How can this strong discrepancy be explained?

Two hypotheses may possibly solve this difficulty:

1. The ice is covered by a black, porous layer with low thermal conductivity. We can see the same for spring snow in towns. The outer surface of such a thin layer absorbs solar energy and transfers it down to the ice boundary, where the temperature is indeed equal to $\sim 200 \mathrm{~K}$. Owing to its porosity, the black layer may be transparent for a gas flow from below. This is not a new idea. The possibility of the presence of such a layer ("mantle") has been discussed for many years (cf. Markovich, 1988). There have been predictions, however, that such a layer would not remain at the relatively small heliocentric distance where the spacecraft encounters with the comet took place.

2. The surface of the nucleus has a horizontally inhomogeneous ("spotted") structure. There are some cold areas with high gas and dust production and some warm ones 


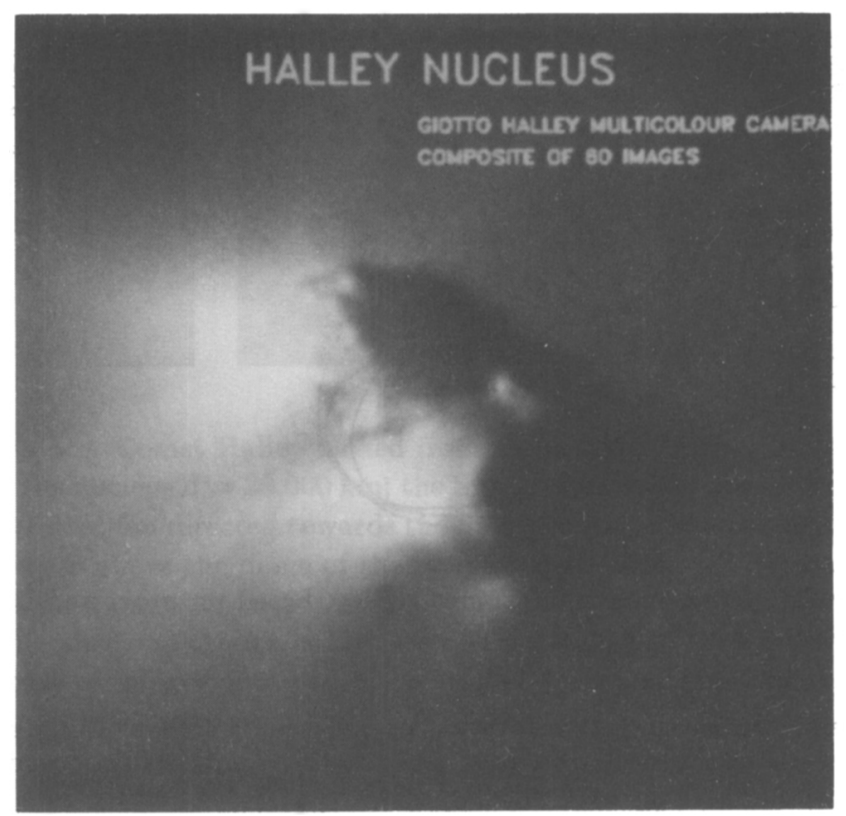

Figure 4: Composite image of the nucleus and the inner coma, as obtained by an analysis of the data obtained by the Giotto Multicolour Camera (Keller et al., 1988). The Sun is to the left. Craters and other surface details are visible near the terminator.

of lower activity. This model has its own difficulties: "open ice", if it exists in cold areas, must be dark. No one has yet presented quantitative evidence that this is really possible. So some specific properties of the surface layer in cold spots are probably implied, even by this model. It is hardly open ice without a more or less pronounced black mantle.

A strong argument for the second model is the thermal lag of about 1.5-2 hours between the regions of the maximal temperature and the subsolar point (Nikolsky et al., 1987). It is not compatible with any reasonable, horizontally homogeneous model.

\section{Neutral and Ionized Gas}

Many cometary molecular and atomic emission lines and bands were recorded during the ground-based observations. But most of the corresponding atoms and molecules (like $\mathrm{H}$, $\mathrm{OH}, \mathrm{C}_{2}, \mathrm{CH}, \mathrm{CN}, \mathrm{NH}_{2}$, etc.) cannot be the actual constituents of cometary nuclei. They are definitely secondary species and they must originate from primary, "parent" molecules. These parent gases, present in a frozen state inside the nucleus, are supposed to be its major constituents. Molecules such as $\mathrm{H}_{2} \mathrm{O}, \mathrm{CO}_{2}, \mathrm{CH}_{4}, \mathrm{NH}_{3}$ and also many other and more complicated ones have been discussed. However, all of these are multiatomic molecules, radiating mainly in the IR range, and therefore not available for observations from the ground.

Studies of Comet Halley by spacecraft provided the real break-through in the solution 
of this difficult problem. Now we have for Comet Halley a long list of actually observed parent molecules (see Table 1). Infrared spectrometers (IKS for $2.5-4.8 \mu \mathrm{m}$ and TKS IR channel for $0.8-1.7 \mu \mathrm{m}$ ranges) were used on the Vega missions and mass-spectrometers (NMS, IMS, PICCA) were on-board Giotto.

Some of the results are presented in Fig. 5. The most intense bands in the IKS range are located near 2.7 and $4.3 \mu \mathrm{m}$. They belong to $\mathrm{H}_{2} \mathrm{O}$ and $\mathrm{CO}_{2}$, respectively. The same molecules were successfully measured by the neutral mass spectrometer (NMS) on Giotto. The results from both experiments are in excellent agreement. They showed that in the gaseous coma, $\mathrm{H}_{2} \mathrm{O}$ is the major constituent among the parent molecules, and that the $\mathrm{CO}_{2} / \mathrm{H}_{2} \mathrm{O}$-ratio is $\sim 3 \%$. The $\mathrm{H}_{2} \mathrm{O}$ band $(2.7 \mu \mathrm{m})$ in the spectrum of Comet Halley was detected already several months before the Vega and Giotto encounters from the highaltitude US Kuiper Airborne Observatory (Weaver et al., 1987); it was observed with extremely high resolving power $\left(\lambda / \Delta \lambda \simeq 10^{5}\right)$.

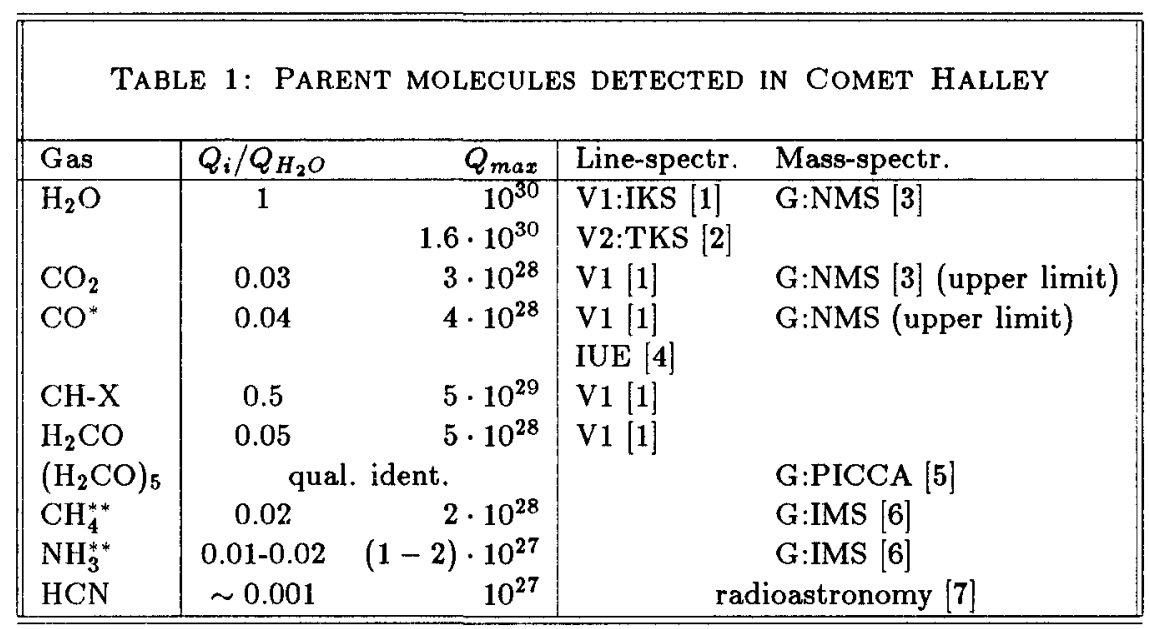

Total $Q_{C O}$ is higher, this is only the part which is directly connected with the nucleus. No direct identification, conclusion is based on IMS data taking into account models. References: [1] Combes et al. (1986); Moroz et al. (1987); [2] Krasnopolsky et al. (1988); [3] Krankowsky et al. (1986); [4] Festou et al. (1986); [5] Hübner, W.F. (1987); [6] Allen et al. (1987); [7] Schloerb et al. (1987).

A broad feature, consisting of a few overlapping peaks, is seen between 3.2 and 3.6 $\mu \mathrm{m}$ (Fig. 5). The central wavelength $3.4 \mu \mathrm{m}$ corresponds to the CH-band in complex organic molecules (hydrocarbons). This feature was confirmed soon after the Vega mission by ground-based observations; fortunately, the terrestrial atmosphere is transparent in this part of the spectrum. A definitive identification of the detected band is not yet possible, but a mixture of saturated and unsaturated hydrocarbons is presumed. Approximate estimates show that their mixing ratio can be larger (up to several tens of percent), if the band is generated in a resonance emission by molecules in the gas phase. However, it is also possible that $3.4 \mu \mathrm{m}$ band is radiated in the process of UV excitation of large molecules or, partly or completely, as thermal radiation from dust grains. 

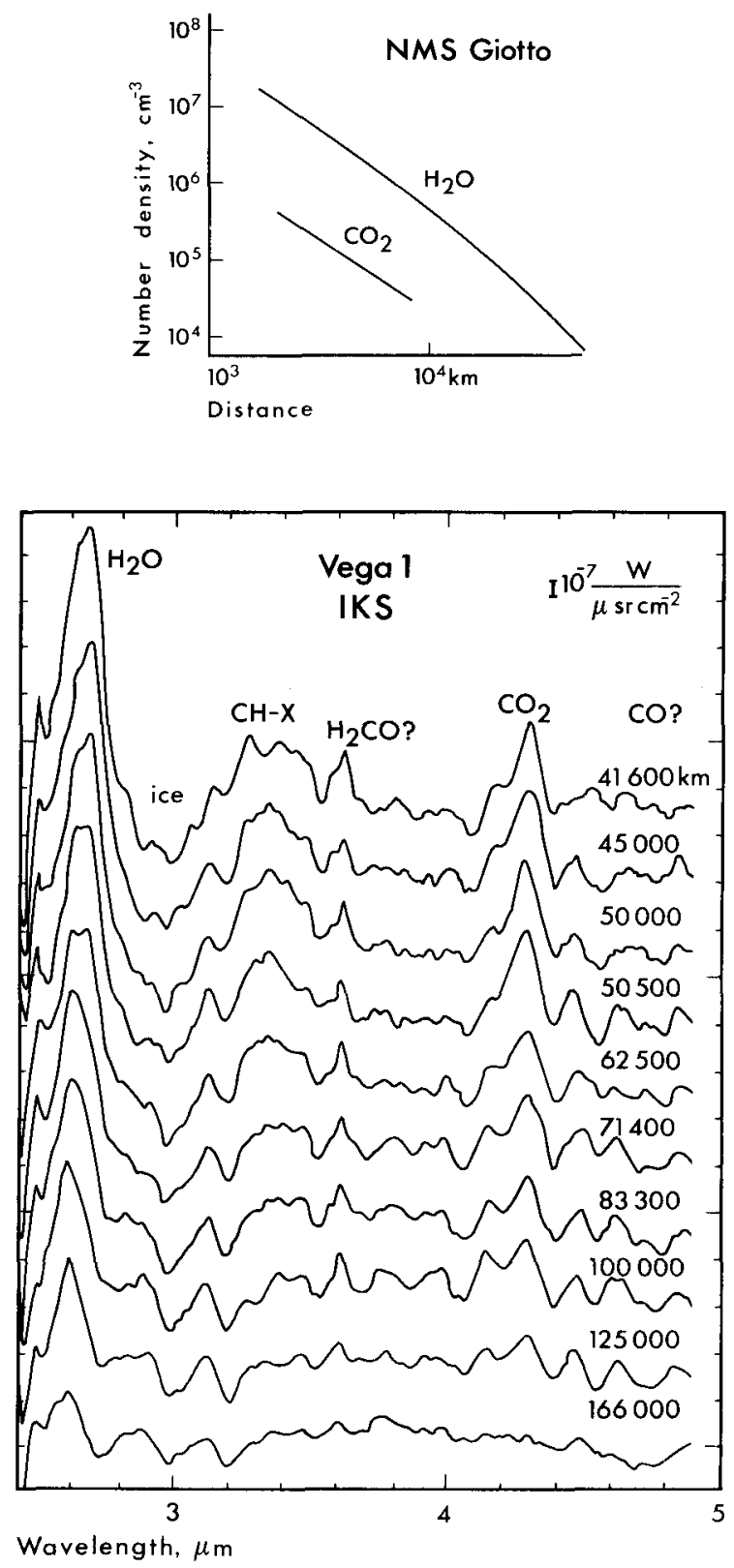

Figure 5 
A narrow peak near $3.6 \mu \mathrm{m}$ has been tentatively identified with formaldehyde $\mathrm{H}_{2} \mathrm{CO}$. According to Hübner (1987), the PICCA mass-spectrometer on Giotto shows the presence of more complicated molecules, including $\mathrm{H}_{2} \mathrm{CO}$ as a part of polyformaldehyde $\left(\mathrm{H}_{2} \mathrm{CO}\right)_{5}$.

The ion mass-spectrometer on Giotto provided many identifications of ionospheric constituents. Taking into account chemical models of the coma, an accurate analysis of the ion data shows that it definitely contains neutral $\mathrm{NH}_{3}$ and very probably $\mathrm{CH}_{4}$ (Allen et al., 1987); both are classical candidates to the list of parent molecules.

Carbon monoxide CO may also be one of the parent molecules. However, part of its flow is generated not by the nucleus itself, but by the so-called "distributed source", i.e. dust or other molecules; in the latter case not all of the CO molecules are parent.

Some of the parent molecules, $\mathrm{CO}_{2}$ and especially $\mathrm{CO}, \mathrm{NH}_{3}, \mathrm{CH}_{4}$, have very low condensation temperatures, much lower than $\mathrm{H}_{2} \mathrm{O}$. Thus, the nucleus of Comet Halley could not have solidified inside the orbit of Neptune. This imposes certain limitations on theories of the origin of comets.

The low condensation temperature, and consequently the high pressure of saturated vapor at small distances from the Sun, could in principle explain the time-variable outburst activity of comets.

The time dependence of the overall gas production rate is now better known for Comet Halley than for any other comet. It shows a strong asymmetry relative to perihelion (Fig. 6). One of the important applications of this dependence is an estimate of the mass of the nucleus. Gas ejected from the nucleus creates a jet force and influences the motion of the comet; in a sense it behaves like a rocket. Rickman (1986) and Sagdeev et al. (1987a, 1988) have independently tried to determine the mass; values from 0.5 to $2 \cdot 10^{17} \mathrm{~g}$ are given by the first, and from 1 to $8 \cdot 10^{17} \mathrm{~g}$ by the second author. The value $2 \cdot 10^{17} \mathrm{~g}$ is within the limits given by both. It corresponds to a density of $0.5 \mathrm{gcm}^{-3}$. The uncertainty is large and several poorly known factors affect this value. However, the average density is most likely lower than 1 and higher than $0.1 \mathrm{gcm}^{-3}$. We can also evaluate a nominal "decay time" for Comet Halley, as $\mathrm{M}$ and $\mathrm{dM} / \mathrm{dt}$ are known; it is $\sim 5 \cdot 10^{4}$ years. In reality, it could be longer, since comets are supposed to gradually reduce their activity in the course of their lifetime.

The neutral gas coma consists of gas that has escaped from the nucleus. It can be treated as a cometary gas atmosphere. Contrary to planets, this atmosphere is not in hydrostatic equilibrium and the number density only obeys the continuity condition. The number density near the surface is $\sim 3 \cdot 10^{13}$ and the pressure is $\sim 1 \mu$ bar. This is equivalent to a good laboratory vacuum. However, the matter in this near-vacuum is still sufficient to generate the entire array of remarkable cometary events.

The outermost part of the cometary atmosphere is its hydrogen coma. It extends over $\sim 10^{7} \mathrm{~km}$, consists of hydrogen atoms and emits the resonance $\mathrm{L} \alpha$ line at $\lambda 1216 \mathrm{~A}$. This hydrogen coma was detected with sounding rockets during earlier observations of other comets. Among all members of the Halley armada, only Suisei was provided with a device for observations of the coma of Comet Halley. These observations commenced about 5 months before the encounter. It was discovered by Suisei that the H-coma is "breathing" and that the intensity varies by a factor of 2 with a 2.2 day period (Kaneda et al., 1986). The explanation is clear: it reflects the rotation of the nucleus and the horizontal inhomogeneity of the surface activity.

Neutral atoms in the coma create ions by means of photoionization. Their number density is low near the nucleus, it increases with distance, passes through a maximum at some distance and decreases further out. The first cometary ions were detected by the spacecraft, $\sim 10^{7} \mathrm{~km}$ from the nucleus.

The interplanetary space is not empty. Solar ions move from the Sun with a speed 


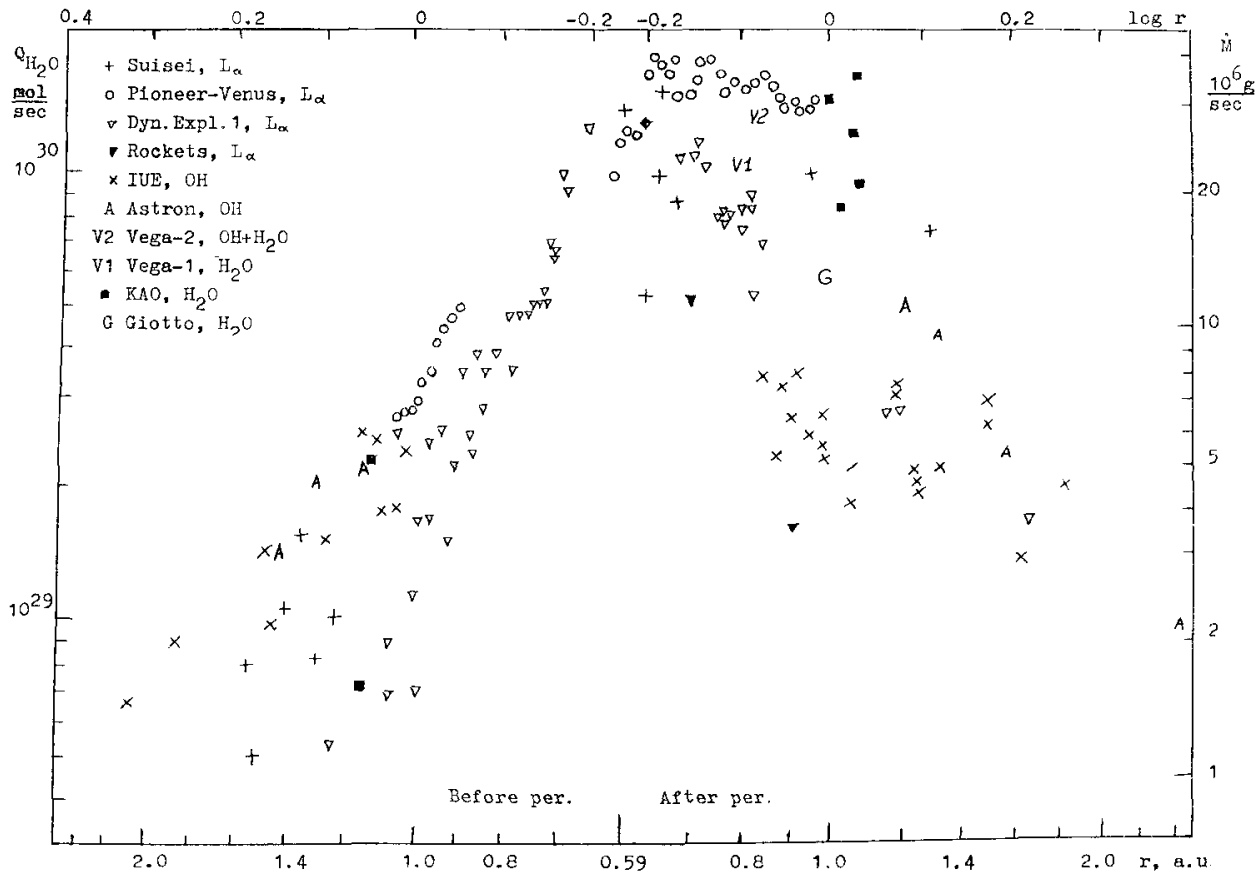

Figure 6: Gas production rate of Comet Halley as function of distance to the Sun before and after perihelion. Abscissa: heliocentric distance; ordinate: gas production rate in mol/s (left scale) and $\mathrm{g} / \mathrm{s}$ (right scale). There is a pronounced asymmetry relative to the perihelion; seasonal effects might be a possible explanation. The data are from flux measurements of three different species: $\mathrm{H}_{2} \mathrm{O}$ [Kuiper Airborne Observatory - Weaver et al. (1987); Vega-1 - Moroz et al. (1987); Vega-2 - Krasnopolsky et al. (1987,1988); Giotto - Krankowsky et. al. (1986)]; OH [IUE - Feldman et al. (1987); Astron - Boyarchuk et al. (1986)]; H [Suisei Kaneda et al. (1986); Pioneer-Venus - Stewart et al. (1987); Dynamics Explorer 1 - Graven and Frank (1987); sounding rockets - Opal et al. (1987)]. 


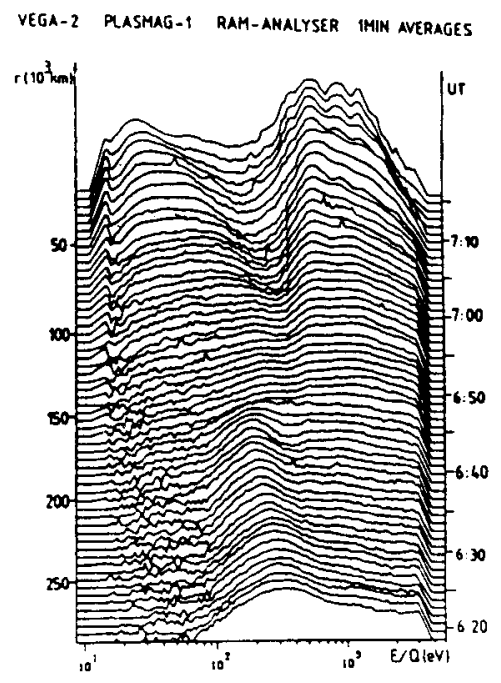

Figure 7: The ion energy spectrum changed during the approach of Vega-2 towards the central region of Comet Halley. The solar wind ions dominate at distances more than $\sim 100,000 \mathrm{~km}$, the cometary ions at smaller distances (Gringauz et al., 1986).

of $400 \mathrm{kms}^{-1}$. This is the "solar wind". Solar ions encounter the ones from the comet. It was expected that a shock wave would arise in this process. Indeed, the spacecraft intersected the shock wave at a distance of $\sim 10^{6} \mathrm{~km}$ from the nucleus. It was "smoother" than predicted by theoreticians. The same property of the shock boundary was observed 6 months earlier by ICE at Comet Giacobini-Zinner.

The speed of the solar wind is diminished, the magnetic field is amplified and a turbulence forms behind the shock wave boundary. The number density of cometary ions increases when we move towards the nucleus. At the distance $\sim 10^{5} \mathrm{~km}$, "quantity converts to quality": within this boundary, the cometary ions dominate in the chemical composition of the plasma (Fig. 7). Such a relatively distinct "chemical boundary" was not predicted by any theory. It has appropriately been named "cometopause". It was observed that the ion flux inside the cometopause is subjected to short-time periodic variations; this phenomenon has not yet been explained.

The next important boundary is the so-called "contact surface" through which the magnetic field does not penetrate (Fig. 8). The contact surface was detected by Giotto at $\sim 5000 \mathrm{~km}$ from the nucleus. The magnetic field dropped from $60 \mathrm{nT}$ to zero when Giotto flew through this contact surface.

\section{Dust}

There is a lot of dust in the coma. A simple explanation of this fact is more or less obvious: the volatiles in the nucleus are mixed with refractory grains and push them outside during the evaporation. Consequently, the measurements of the composition of the dust grains have told us about the nature of the refractory material inside the nucleus.

New and important information about this subject was obtained by the dust impact mass-spectrometers PUMA on Vega (Sagdeev et al., 1987) and PIA on Giotto. A target 


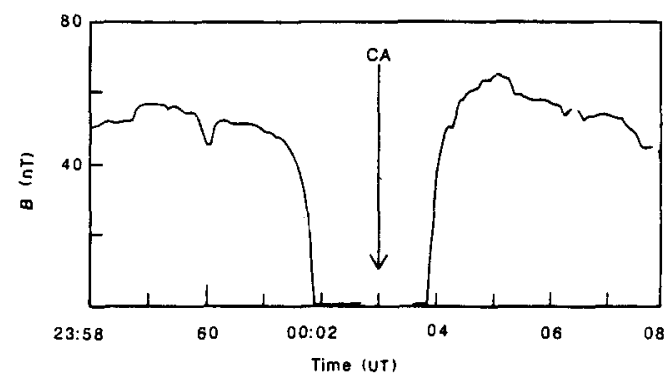

Figure 8: Magnetic "hole" inside the comet (Neugebauer et al., 1986)

of chemically pure silver was placed at the entrance of the mass-spectrometers. A dust particle converts to a small cloud of ions when impacting on this target. The ion cloud enters the mass-spectrometer, allowing the measurement of its elemental composition. Only small dust grains with the masses in the $10^{-16}-10^{-14} \mathrm{~g}$ interval were studied in this way, but there were many of them. For example, PUMA registered about 2000 cometary dust particles.

There were particles with predominance of light elements, carbon or metals. Sometimes peaks of $\mathrm{H}, \mathrm{C}, \mathrm{O}, \mathrm{N}$ were visible simultaneously. The analysis of these so-called CHON particles led to the next interesting conclusion: 1) these particles have a $\mathrm{C} / \mathrm{Si}$ relative abundance which is substantially higher than in any meteorites of known classes; 2) nitrogen is present in all particles with large $\mathrm{C} / \mathrm{Si}$-ratio, but this is possible only if both $\mathrm{C}$ and $\mathrm{N}$ are constituents of organic molecules. Thus we have here the second evidence that there is organic matter within the cometary nucleus. Greenberg qualitatively predicted the existence of CHON particles on the basis of his model, describing the nucleus as a "snowball" that consists of interstellar dust grains. He favoured a complicated structure of such grains (see below).

The existence of CN-jets correlated with dust (ground-based observations) is probably explained by the outgassing of CHON particles. This correlation between gas and dust is one of the hitherto unknown cometary properties which were detected during this apparition of Halley.

Many other, less complicated particle detectors were used during the cometary missions in order to measure in-situ number densities and mass distributions - on Vega (Masets et al., 1987; Vaisberg et al.; 1987, Simpson et al., 1987) and on Giotto (McDonnell et al., 1987). The number of small grains is higher than what was predicted from earlier ground-based remote observations. A summary of the physical properties of cometary dust is given in Table 2. There is evidence of destruction of dust particles on their paths. It means that at least the larger particles are fragile. Their density is probably much less than $1 \mathrm{gcm}^{-3}$. Many examples of fragile, fine-structured interplanetary particles (so-called Brownly particles) have been collected in the terrestrial stratosphere. Their cometary origin is now almost proven. A direct calibration of the dust particle detectors was not possible and for this reason the estimates of the dust production rate for Comet Halley can only be given with an accuracy of half an order of magnitude. The dust-to-gas ratio was probably between 0.1 and 0.5 at the time of the Vega and Giotto encounters. 


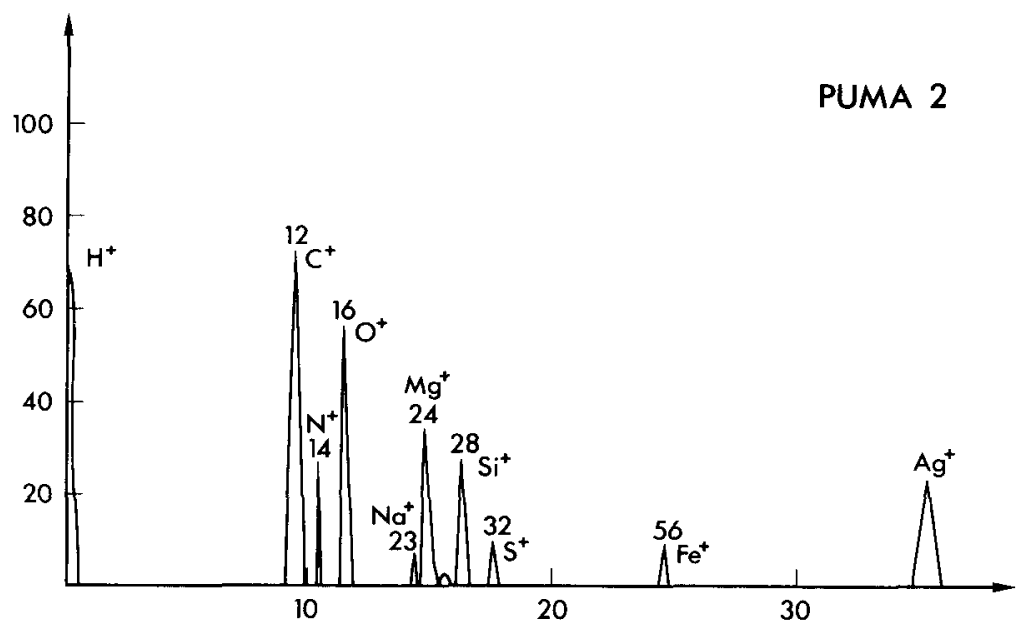

Figure 9: One of the mass-spectra obtained by the particle impact mass-spectrometer PUMA on Vega-2. This example shows that a complicated mixture of silicate, metallic and organic material is present in some cometary particles.

TABle 2: Some Propertes of COMEtary Dust

(a) Cumulative mass distribution: $\mathrm{r}=8100 \mathrm{~km}$ (Vega-2)

$$
\begin{array}{ll}
3 \cdot 10^{-16}<\mathrm{m}<10^{-10} \mathrm{~g} & \mathrm{~F}(>\mathrm{m}) \propto \mathrm{m}^{-0.3} \\
10^{-10}<\mathrm{m}<3 \cdot 10^{-7} \mathrm{~g} & \mathrm{~F}(>\mathrm{m}) \propto \mathrm{m}^{-0.85}
\end{array}
$$

(b) Optical "activity"

$90 \%$ of scattered solar light is provided by particles with $\mathrm{m}>10^{-12} \mathrm{~g}$

(c) Average density of particles with $\mathrm{m}>10^{-12} \mathrm{~g}$

(d) Velocity

$$
\sim 0.3 \mathrm{gcm}^{-3} \quad \text { uncert. factor } \sim 2
$$

$$
\begin{array}{cc}
\mathrm{m}(\mathrm{g}) & \mathrm{u}_{d}\left(\mathrm{krns}^{-1}\right) \\
10^{-12} & 0.75 \\
10^{-8} & 0.5 \\
\hline
\end{array}
$$

a - Masets et al. (1987); b,c,d - Krasnopolsky et al. (1987) 


\section{Concluding remarks}

\section{The nucleus}

Let us now summarize what we know about the nucleus of Comet Hally, taking into account the information obtained by the studies of gas and dust. It is a single solid body; it cannot be a collection of gravitationally bound or unbound small components. Water ice is the main volatile constituent. There are many other, less abundant constituents, the most important of them are probably organic molecules as in volatiles and/or refractory compounds. The average density of the nucleus is low, probably less than $1 \mathrm{gcm}^{-3}$.

A model of the cometary nucleus as a single, solid body consisting of a mixture of volatiles and meteoritic matter was proposed by F. Whipple (1950). His model has been confirmed by the Halley missions. In addition, some new important properties were found:

1. the presence of the organic matter in different forms,

2. a pronounced inhomogeneity of the surface properties,

3. a low albedo,

4. a high temperature in some areas,

5. specific properties of the surface layer, and

6. a low density.

Different ideas about the internal structure of the nucleus are now being discussed and two of them will be mentioned here. The first was elaborated by Greenberg (1982) who described the nucleus as a package of interstellar grains. These are supposed to be sr.all cylinders, a few microns in length. Each of them contains all the components of the nucleus: silicate core, organic internal mantle, an icy external mantle and even submicron particles within the latter.

But this is only one of the hypotheses under discussion. Another example is an icy glue model proposed by Gomboshi and Houpis (1986). It describes the nucleus as a collection of many massive silicate stones connected by ice layers of varying thickness. This concept explains very well the linear shape of the active regions on the surface of the nucleus. There are other models, but these two are probably the extreme cases. We could hardly decide between them without cometary sample return missions. Such plans are discussed very enthusiastically in the wake of the successful Halley missions. This is one of the most important new lines in the future studies of the Solar System.

\section{The place of the comets in the Solar System.}

Comets almost definitely come from Oort's cloud. It contains $10^{11}-2 \cdot 10^{12}$ cometary nuclei and is located at $2 \cdot 10^{4} \mathrm{AU}$ from the Sun. It is the most distant component of the Solar System. If the mass of Comet Halley's nucleus is typical and if the number of such nuclei in Oort's cloud is $2 \cdot 10^{12}$ then its mass is $\sim 100 \mathrm{M}_{\oplus}$, i.e. $1 / 4$ of the total mass of all planets. The angular momentum could then be of the order of one magnitude higher than that of the planets. These two simple ideas were mentioned recently by Marochnik and Mukhin (1987). They lead to a new understanding of the possible place of comets in the Solar System. It may be much more important than was supposed before. 


\section{References}

References to papers presented at the 20th ESLAB Symposium on the Exploration of Halley's Comet, Heidelberg, eds. by Battric B., Rolf, E.J., Reinhard R., ESA SP-250, Noordwijk, The Netherlands, ESA Publ. Div., 1986, are marked (H). Most of the papers presented at this symposium were also published in Astron. and Astrophys., Volume 187. In these cases references are given to the appearance in this journal.

A'Hearn, M.F., 1988, Ann. Rev. Earth Planet. Sci., 16, 273

Allen, M. et al., 1987, Astron. and Astrophys., 187, 502

Boyarchuk, A.A. et al., 1986, (H), Vol.3, 193

Combes, M. et al., 1986, Nature, 321, 266

Feldman, P. et al., 1987, Astron. and Astrophys., 187, 325

Festou, M.C. et al., 1986, Nature, 321, 361

Festou, M. et al., 1987, Astron. and Astrophys., 187, 575

Galeev, A.A., 1987, Astron. and Astrophys., 187, 12

Gomboshi, T.I. and Houpis, H.L.F., 1986, Preprint KFKI-1986-23/C, Budapest

Graven, J.D. and Frank, L.A., 1987, Astron. and Astrophys., 187, 351

Gringauz, K.I. et al., 1986, Geophys. Res. Lett. 13, 613

Greenberg, J.M., 1982 in Comets, ed. Wilkening, L.L., Tucson, University of Arizona Press, 131

Hübner, W.F., 1987, Science, 237, 628

Kaneda, E. et al., 1986, Geophys. Res. Lett., 13, 833

Keller, H.U. et al., 1986a, Nature, 321, 320

Keller, H.U. et al., 1986b, (H) Vol.2, 347

Keller, H.U. et al., 1988, Nature, 331, 333

Kissel, Y. et al., 1986, Nature, 321, 336

Krankowsky, D. et al., 1986, Nature, 321, 326

Krasnopolsky, V.A. et al., 1987, Astron. and Astrophys., 187, 707

Krasnopolsky, V.A. et al., 1988, Astron. and Astrophys. (in press)

Markovich, M.Z., 1958, Bull. of Inst. of Astrophys. and Phys. (Dushana, USSR), 28, 25 (in Russian)

Marochnik, L.S. and Mukhin, L.M., 1987, Preprint IKI Pr-1319, Moscow (in Russian)

Moroz, V.I. et al., 1987, Astron. and Astrophys., 187, 513

Millis, R.L. and Schleicher, D.G., 1986, Nature, 324, 646

Neugebauer, F.M. et al., 1986, Nature, 321, 352

Nikolsky, Yu.V. et al., 1987, Kosmich. issled., 25, 793 (in Russian)

Opal, C.B. et al., 1987, Astron. and Astrophys., 187, 320

Rickman, H., 1986, Proc. of an ESA Workshop held at the University of Kent at Canterbury, UK, 15-17 July 1986, xxx

Sagdeev, R.Z. et al., 1986a, Nature, 321, 262

Sagdeev, R.Z. et al., 1986b, (H) Vol.2, 307

Sagdeev, R.Z. et al., 1986c (H) Vol.2, 335

Sagdeev, R.Z. et al., 1987a, Pisma v Astron. Zh., 13, 621 (in Russian)

Sagdeev, R.Z. et al., 1987b, Kosmich issled., 25, 856 (in Russian)

Sagdeev, R.Z. et al., 1988, Nature, 331, 340

Schloerb, F.P. et al., 1987, Astron. and Astrophys., 187, 47

Sekanina, Z. and Larson, S.M., 1986, Nature, 321, 357

Stewart, A.I.F. et al., 1987, Astron. and Astrophys., 187, 351

Weaver, H.A. et al., 1987, Astron. and Astrophys., 187, 411

Whipple, F., 1950, Astrophys. J., 111, 375 